\title{
Context-Aware User Interface Framework for Mobile GIS
}

\author{
Jiangfan Feng and Yanhong Liu \\ College of Computer Science and Technology, Chongqing University of Posts and \\ Telecommunications, Chongqing 400065, China
}

fengjf@cqupt.edu.cn

\begin{abstract}
Although mobile phones currently are taking Geographic Information Systems (GIS) out of the office and into the field, human-computer interaction $(\mathrm{HCI})$ as a field is not yet theoretically equipped to match this reality. Consequently, human-GIS interface provided by mobile phones makes the growing influence on residents, they obtain related services through submit their request like search routes. However, the information offered by users is limited. To get more hidden information can make the command of users accurately; therefore, context-aware is advocated. In this article, we proposed a method which considering the existing context to concern users' ability and combining with the user feedback to provide a more effective way of human-GIS interface on mobile phones. Through a design case, we develop the methodological approach and illustrete how the model can be applied to analyze the context of users.

Keywords: Geographic Information Systems (GIS), Human-Computer Interaction (HCI), User Interface, Context-aware
\end{abstract}

\section{Introduction}

Today moble devices can compete with desktop computers and laptops in providing applications and service in manly situations. All of these devices may not necessarily have a well specified function in darly use; rather, they are used interchangeably in response to the context and conditions of use. Human-GIS interface provided more service for residents gradually owing to the rapid growth of mobile terminal such as mobile phones. And in order to achieve a befter interaction, context used (e.g., location or preference of the users, existed experiments the surrounding environment), in doing so, they understand the command of users accurately and also reduce users' input. In this case, users who are often played an internediate role, they make a choice on the context information which is collected by sensors, and then tell the applications that they want to choose. The need for interactions between mobile devices and users has introduced a new concept called context-aware [1].

Recently, many researchers are trying to take the advantages of context to solve problems especially Human-GIS interaction. Besides, problems in this area can utilize knowledge of users' current location information obviously. Gregory D. Abowd and Christopher G. Atkeson et al. proposed a mobile context-aware tour guide in 1997 [2], GuorayCai specify a semantic model which combined with context and demonstrate how this model supports contextualized interpretation of vague spatial concepts during human-GIS interactions in 2007 [3], Jaewoo Chung, Paulina Modlitba and Chaochi Chang proposed a mobile user-aware route planner which can learns a user's everyday routes and provides directions from locations along those routes in 2008 [4], Namgyu Kim et al. developed an application named Optimize Your Time (OYT, for short) which committed to take the shortest time to reach the specified destination 
for users in 2009 [5], Neal Lathia and Licia Capra et al. discuss a multi-layered approach to learn travelers' surrounding environment and using these context to provide a best traveling proposal in 2012 [6]. And generally speaking, context based on mobile phones can be divided into three parts as follows [7]:

(i) User environment: location, preference, experiment, social relations etc.

(ii) Mobile environment: device suitable for users to input or display, network, Bluetooth, etc.

(iii) Physical environment: weather, date, noisy, etc.

In summary, context has an increasingly impact based on the mobile terminal, and shows the superiority of increasing information since the implicitly indicated by the user.

Location currently, destination the user possibly wanted and the kind of transport may be selected and have become the greatest impacts of context: they formed the key elements of the route. The main limitation of this approach is that many other factors are ignored. For example, in many cases such as medical treatment, users often prefer to go to a fixed hospital for appointing the same doctors. When user requests this service, system can record the information of the user as his tendencies. If we record the choice he makes and the frequency of the similar decision, then we can provide a detailedoprogram to reach the hospital according to their preference or special cireunstance next Yime. For instance, the patient wants to change a hospital or the doctor rest. Syster gan remind the patient and provide reasonable suggestions. We pay more attention to users themselves than the route finding, eliminate their thinking time and help them to make a quick choice. The reminder of this paper contains a detailed description of this ideta, as well as a result of the evaluation.

In this paper, we proposed method to integrate the available context and combining with the users' feedback to filten the context invakuable. This will be followed by a description of the context-aware of the problem and a detailed presentation of how the required evaluation method is defined.

\section{Related Works}

\subsection{Mobile Sensors}

Mobile phone plays an important role in people's daily lives already as a portable integrated terminal, which not just provides communication service, but contain a rich set of sensors (e.g., camera, accelerometers, GPS, digital compass, gyroscope, ambient light sensors, proximity sensors, multi-touch panels, microphone) [8]. These sensors integrated with mobile phones are videly used to provide users with many novel applications through perceiving the environment around users. Take a few examples: an energy efficient routing scheme might be found by using GPS which can get the location information and the energy levels of sensor nodes [9]. It is easier to learn users' surroundings when the camera and microphone used. Furthermore, Wi-Fi or wireless network can provide a suitable service based on users' interest or social networks on a map [10]. In addition, light sensors, temperature sensors can be perceptions of the users' environment in which the tools provide more detailed context.

Following the trend of "people-centric sensing", people who carry the sensor device and benefit from the information obtained get more attention than sensors [11-12]. Their patterns of interaction with a mobile device, some things of interest, events are collected for themselves or others to make choices. In the era which filled with mobile terminal, people-centric sensing will help us linked more closely by share and collect information related to us. And the user is one of the most important elements, they flow in public places 
and interact with others, they also affect the development of event around. In addition, public sensing may share much useful information for public benefit [11]. There some projects which are related to people-centric sensing are ongoing, and the sensing capability of mobile phones is getting stronger like APPLE iPhone.

\subsection{Context-aware}

When in a process of human-human conversation, implicit information often used to rich the content they share. It's seems natural when human utilized because they may understand the situation each other, but it's difficult to make a computer master this skill. Recently, there is not necessary to find out everything about users through asking them. Otherwise, we take the advantages of context to increase the richness of interaction and provide usepulservice. However, the definition of context is different to different people. Context-awarelapplication existed since 90s, and Schilit and Theimer [13] put forward that context contains location, identities of nearby people and objects in 1994, Brown and Bovey [14] added time of the day, season of the year and temperature to the original definition in 1997 To the present, context has a broader meaning that added much related information. For instance, if we know the location of a person, we can get the surrounding environment of him such as how far to the nearest restaurant or how about the weather, even what happening around him.

Context-aware systems would collect confexy information related to users, and service for them. Besides, context-aware is the ability to sense, detect and grab the environment around users' location, get the dynamic changes from the location and even speculate their behavior based on the context we obtained [ [5] Schilit 13 ] proposed that context-aware computing can provide us with a display of locating an object, nearby devices which can be used for us, detecting of nearby people and service which are relevant to reminders. Anind K. Dey [16] put forward that context-aware also contans information presented, users' service executed automatically and tagging the context information so as to next service. Dey and Abowd [7] proposed that "a system is context-alvare if it uses context to provide relevant information and/or services to the user, where relevancy depends on the user's task". In the generally interaction, people input information by keyboard, touch screen, microphone etc. While context-aware aim to reduce users' input combined with vivid kinds of mobile sensors, human-computer interaction' can be more humanized. At the same time, context-aware not just location when cooperate with geographic information system (GIS), it can enrich the function of GIS and provide to users a variety of services.

\subsection{Smart Phone Interfaces}

We cannot only use a mobile phone to call others or send messages, but also use a mobile phone to do some searching and so on. In the process, we contact with interfaces, of a device is the look and feel of the on-screen menu system. For example, how it works, its style scheme, how it feedback to users, all of these things are part of the user interface. It is also used to refer to the piece of software that resides on a smart phone that deals with typical user interface issues. Such as Nokia S60 is one of a user interfaces that run on the Symbian OS on smart phones.

For different people, interfaces may change for one interface cannot suitable for all people. Many researchers proposed sundry interfaces based on users' ability. Sulaiman and Sohaimi [18] discuss a possible interface which is simple enough for older users through analyzing the situation of using a mobile phone. Xiaowei Cao and Sri Kurniawan [19] put forward a mobile phone interface for children. All of these are committed to reach a better human-computer 
interaction, then resulting in the concept of smart interface, which aims to achieve better interaction by using environment surrounded and users' interests.

\subsection{Routing Problem}

People often need tools to find routes in daily lives, and mobile phone becoming an optimal choice for their easy to carry, ability of computing and sensing capabilities. In the problem of route choice, users usually hope to easily decide where they go, how they reach and what they do in the way. To reach these goals, we need mobile sensors to collect context information, and context-aware applications to analyze users' intention accurately. Some users want to get a fastest route in their trip, but some want to get a suitable route to enjoy the view in the travel process. An environment friendly route to meet users is different yo different individuals. Besides, about the choice of transport (e.g., bus, foot, car, train) always related to these: lowest cost, fastest way, traffic conditions, lowest emission and so on.

There is a great quantity researches of route finding. Lathia and Capra (6) proposed mobile travel information services based on a multi-layered approach. Witteveen [20], etc. put forward an algorithm to finding a conflict-free schediule along a fíxed path. Personal route can also predict through context and then provide a similar route. Mazhelis and Zliobaite [21] proposed context-aware route recognition approach to improve the accuracy of routing recognition. In this brief review, these route findings both using context to increase the access to information, the seeking of route not enly needs the consideration about personalization, but also needs the group to share infonnation, which decided by the individual and common of a person.

\section{Adaptive Spatial Information}

Human-GIS interfaee should have a certain degree of adaptive capacity, let the spatial information dynamically generated based on the user's preference, the variable of the given task, location, eto. The expression of spatial information has four following factors: interactive en ironment, user baekground, user orientation, carrying capacity (shown in Figure 1).

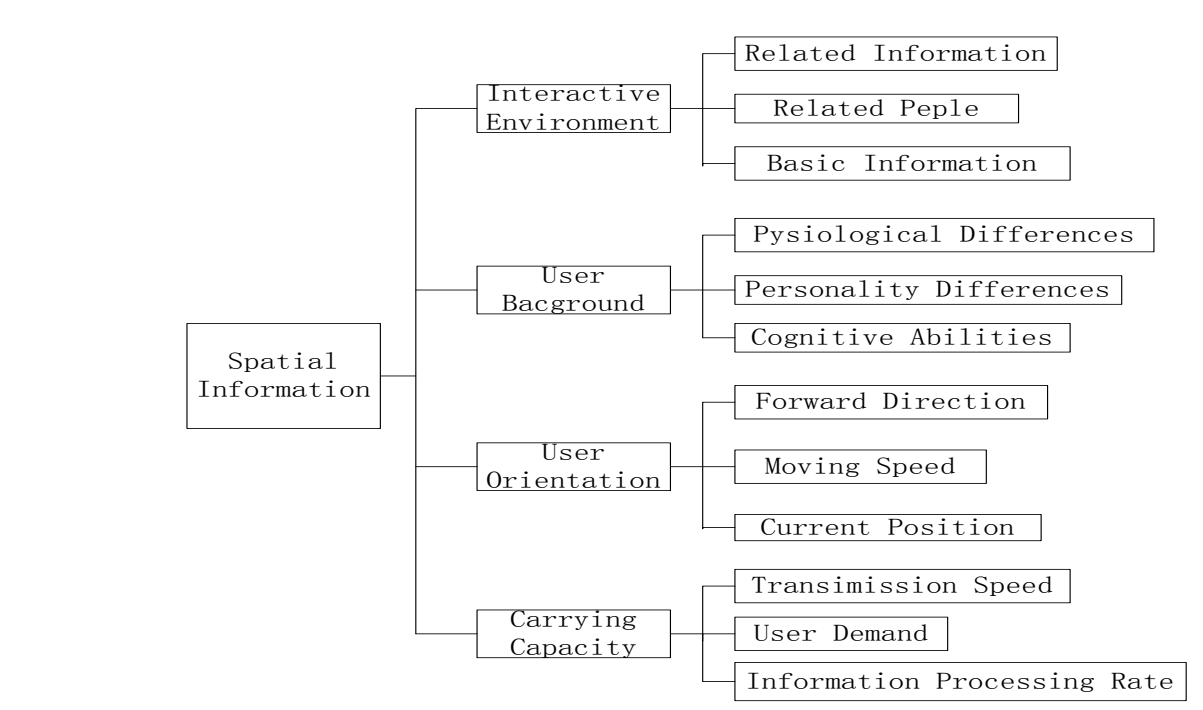

Figure 1. Context Classification 
(1) The factors affecting the interactive environment: basic information which is included weather, date, time, etc., related information means that the information around the user and related to user's task. Related people mean that the information of the other users who are associated with the user's task.

(2) The major influencing factors of user background are cognitive abilities, personality differences and physiological differences. Three factors can be abstracted into: the degree of cognitive, variables of physiological differences, personality differences.

(3) The user's location can be abstracted into: current position, velocity parameters, andothe direction vector. These three quantities are changing with time, We represen them respectively in the following form: $\mathrm{p}, \mathrm{v}, \mathrm{r}$. means that the prediction the location of the user appears in less time and then let the map shows this area so if can reached the map dynamic matching effect as in Figure 2.

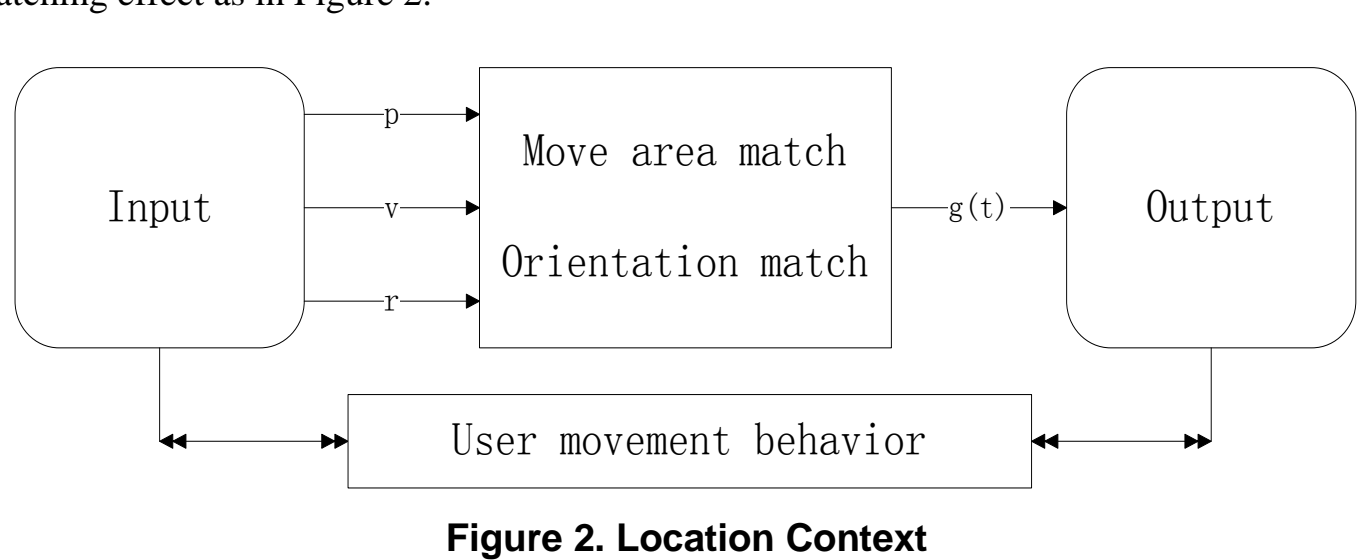

\section{Experiments and Analysis}

\subsection{Context of the Interface Elements}

Human, hardware and software, the data is computerized system is a three while the interface is linked oond of the three. The flow of information from data to human as important as the flow of information from human to data. These two aspects of the information smooth flowing are(important goals of adaptive human-computer interaction. We often need to focus on the context information given by the user, for example, in the same time the user will find the route and also check the weather. There are weather tips given automatically if the user always to check as in Figure 3 (left image). In addition, different users have different capacities, for example, aged users often need to enlarge the font then they can see clearly, we do the operation automatically to enlarge the font though get the users' age as in Figure 1 (right two images). 

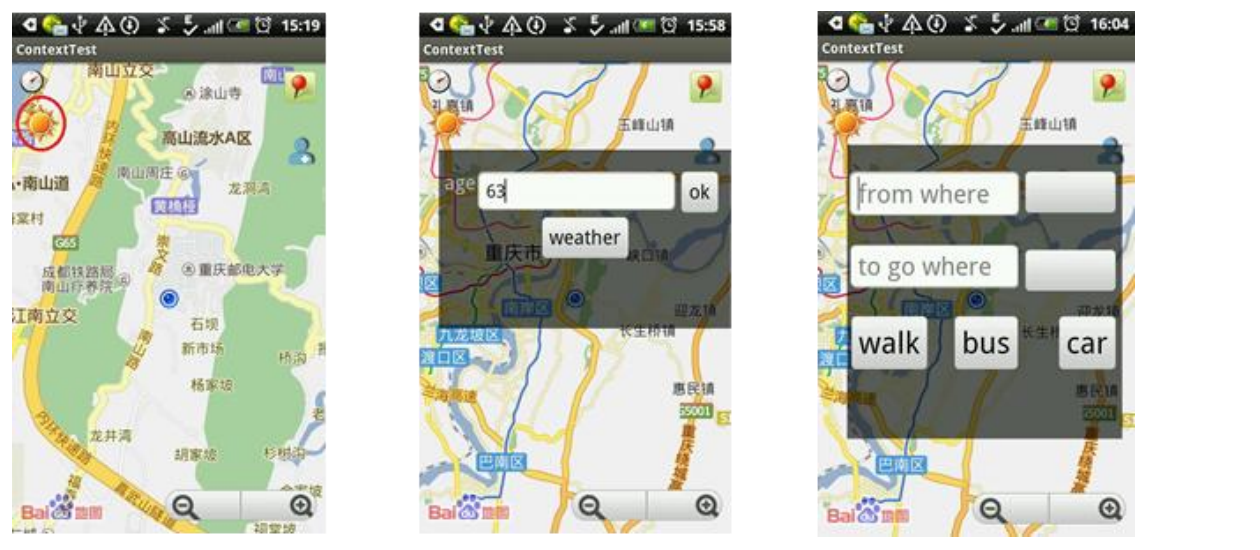

\section{Figure 3. Weather-related and Age-related Context of Interface}

\subsection{Context of User Orientation}

User's orientation contains location, speed and direction. The expression of the spatial information, often uses these to make an adaption of the map display area or the map display orientation. In addition, in order to reduce the input, the orientation of the user in the past can serve as a context for the user by way of marking, so the user can easily access.

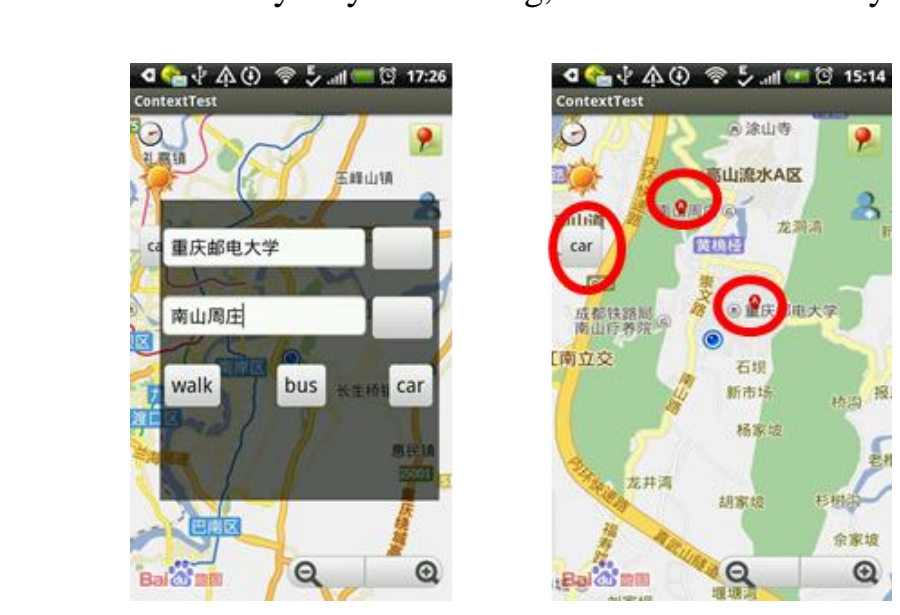

Figure 4. Context of Users' Location

When the user chooses the same place for a certain time, the map will give a red marker, this icon allows the user chose directly. The means of transport choices have the same situation.

\subsection{User Evaluation and Analysis}

After summarizing, we proposed four factors that affect the user experience which are adaptive, context utilization, interface friendliness and operating convenience. We were used A, U, F, C to represent these factors. By the way of user investigation, we calculated the weight of these variables. 
Table 1. Influencing Factors and Weights

\begin{tabular}{cllll}
\hline Test & A & U & F & C \\
\hline 1 & 0.2 & 0.2 & 0.2 & 0.4 \\
2 & 0.2 & 0.3 & 0.3 & 0.2 \\
3 & 0.4 & 0.2 & 0.1 & 0.3 \\
4 & 0.3 & 0.3 & 0.2 & 0.2 \\
5 & 0.3 & 0.2 & 0.3 & 0.2 \\
Weight & 0.28 & 0.24 & 0.22 & 0.26 \\
\hline
\end{tabular}

We use the Mapbar to do the comparison experiment for the navigation application Can on behalf of the most such navigation software. We select users that parts of them are familiar with map operation, but the others are not. In the user-assessment questionnaire, we define the evaluation grade is: excellent, good, normal and poor, and quantify these four levels are: 1, $0.8,0.6,0.4$. Table 2 shows the evaluation of users to Mapbar, and Table 3 shows the evaluation of users to the test in this paper.

Table 2. Mapbar-Assessment

\begin{tabular}{|c|c|c|c|c|}
\hline Test & A & 0 & $\mathrm{~F}$ & $\mathrm{C}$ \\
\hline 1 & 0.8 & a & 0.8 & 0.6 \\
\hline 2 & 0.4 & & & 0.6 \\
\hline 3 & 0.6 & 0.6 & 0.8 & 0.4 \\
\hline 4 & 0.8 & 0.6 & 0.8 & 0.8 \\
\hline 5 & 0.6 & 0.4 & 1 & 0.6 \\
\hline 6 & 0.8 & 06 & 0.8 & 0.8 \\
\hline 7 & & 0.8 & 0.6 & 0.4 \\
\hline 8 & & 0.6 & 0.8 & 0.8 \\
\hline 9 & & 0.8 & 0.8 & 0.6 \\
\hline 10 & 0 & 0.8 & 1 & 0.6 \\
\hline Tester & & $\mathrm{U}$ & $\mathrm{F}$ & C \\
\hline 1 & 0.8 & 0.6 & $\begin{array}{l}0.6 \\
\end{array}$ & 0.8 \\
\hline 2 & 0.6 & 0.8 & 0.6 & 0.8 \\
\hline 3 & 0.8 & 1 & 0.6 & 0.6 \\
\hline & 0.8 & 0.8 & 0.4 & 1 \\
\hline & 0.8 & 0.8 & 0.8 & 0.8 \\
\hline 0 & 1 & 0.6 & 0.6 & 0.8 \\
\hline 7 & 0.6 & 0.8 & 0.6 & 0.8 \\
\hline 8 & 0.8 & 0.8 & 0.6 & 1 \\
\hline 9 & 0.8 & 0.8 & 0.4 & 0.8 \\
\hline 10 & 0.8 & 0.8 & 0.4 & 0.8 \\
\hline
\end{tabular}

We use the Fuzzy Evaluation Method, factor set $M=\{A, U, F, C\}$, evaluation level V= $\{\mathrm{E}, \mathrm{G}, \mathrm{N}, \mathrm{P}\}$, weight of the factors $W=\left\{w_{1}, w_{2}, w_{3}, w_{4}\right\}$ (meet $\sum_{i=1}^{n} w_{i}=1$ ), $B=W R=\left(\overline{b_{1}}, \overline{b_{2}}, \ldots, \overline{b_{m}}\right)(\mathrm{R}$ on behalf of the matrix which user evaluate based on several factors). 
We can get $B_{\text {Mapbar }}=(0.7,0.592,0.592,0.752,0.64,0.752,0.596,0.696,0.748,0.736)$ and we can get $B_{\text {Test }}=(0.708,0.7,0.752,0.764,0.8,0.764,0.7,0.696,0.748,0.736)$. The average values of four factors assessed separately are $\overline{M_{\text {Mapbar }}}=\{0.1848,0.1584,0.1628,0.1612\}$ and $\overline{M_{\text {Test }}}=\{0.2296,0.1872,0.1232,0.2132\}$ By contrast, we can find context using and adaptability are also improved.

\section{Conclusion}

In this paper, we have presented the context application on Human-GIS interface and proved the advantages of this paper method on the route searching. The main contribution is that it minimizes user input and adapts to the user characteristics. In the near feature, we may strive to excavation more contexts to make the Human-GIS interface moreadaptive.

As a future work, we will propose the enhancement of interaction framework; it should not have a specific place in a design process but can be used throughout design.

\section{Acknowledgments}

The work is supported by the National Naure Science Foundation of China (No. 41101432, 41201378), the Natural Science Foundation Project of Chongqing CSTC (cstc 2012jjA40014).

\section{References}

[1] N. Kim, H. S. Lee, K. J. Oh and J. Y. Choi, "Confext-aware mobile service for routing the fastest subway path", Expert Systems with Applications, vel. 36. n\%. 2, (2009).

[2] G. D. Abowd, C. G. Atkeson and J. Hong "Cyberguide: A mobile context-aware tour guide", WIRELESS NETWORKS, vol. 3, no. 5, (1997).

[3] G. Cai, "Contextualization of Geospatial Database Semantics for Human-GIS Interaction", GEOINFORMATICA, vol. 11, no 2, (2007).

[4] J. Chung and C. Sehmandt, "Going My Way: a user-aware route planner. Proceeding of the SIGCHI Conference on Human Factorsin Computer Systems", (2009), New York, USA.

[5] N. Kim, H. S. Lee, K. J. Oh and J. Y. Choi, "Context-aware mobile service for routing the fastest subway path”, Expert Systems with Applications, vol. 36, no. 2, (2009).

[6] N. Lathia, L. Capra, D. Magliocchetti, F. De Vigili, G. Conti, R. De Amicis, T. Arentze, J. Zhang, Davide Cali and V. Alexa, Personalizing Mobile Travel Information Services", Transport Research Arena, vol. 48 (2012).

[7] G. D. Abowd A. K. Dey, P. J. Brown, N. Davies, M. Smith and P. Steggles, "Towards a Better Understanaing of Context and Context-Aware", Handheld and Ubiquitous Computing Lecture Notes in Computer Seience, (1999) September 27-29, Germany.

[8] D. Che N. D. Lane, T. Tsung-Te Lai, C. Pang, X. Meng, Q. Guo, F. Li and F. Zhao, "Balancing Energy, Latency and Accuracy for Mobile Sensor Data Classification", Proceeding of the 9th ACM Conference on Embedded Networked Sensor Systems, (2011), New York, USA.

[9] K. Kim, J. Yun, J. Yun, B. Lee and K. Han, "A location based routing protocol in Mobile Sensor Networks", Advanced Communication Technology, (2009) February 15-18, Phoenix Park.

[10] S. Gaonkar and R. R. Choudhury, "Micro-Blog: Map-casting from Mobile Phones to Virtual Sensor Maps", Proceeding of the 5th international conference on Embedded networked sensor systems, (2007) November, New York, USA.

[11] A. T. Campbell, S. B. N. D. Lane, E. Miluzzo, R. A. Peterson, H. Lu, X. Zheng, M. Musolesi, K. Fodor and G.-S. Ahn, "The Rise of People-Centric Sensing", Internet Computing, IEEE, vol. 12, no. 4, (2008).

[12] E. Miluzzo, N. D. Lane, K. Fodor, R. Peterson, H. Lu, Mirco Musolesi, S. B. Eisenman, Xiao Zheng and A. T. Campbell, "Sensing Meets Mobile Social Networks: The Design, Implementation and Evaluation of the CenceMe Application. Proceedings of the 6th ACM conference on Embedded network sensor systems, (2008), New York, USA. 
[13] B. N. Schilit and M. M. Theimer, "Disseminating Active Map Information to Mobile Hosts", vol. 8, no. 5 (1994).

[14] P. J. Brown, J. D. Bovey and X. Chen, "Context-Aware Applications: From the Laboratory to the Marketplace", Personal Communications, vol. 4, no. 5, (1997).

[15] W. Liu, X. Li and D. Huang, "A Survey on Context Aware", Computer Science and Service System (CSSS), (2011), June 27-29, Nanjing, China.

[16] A. K. Dey, "Understanding and Using Context", Personal and Ubiquitous Computing vol. 5, no.1, (2001).

[17] S. Sulaiman and I. S. Sohaimi, "An Investigation to Obtain a Simple Mobile Phone Interface for Older Adults", Intelligent and Advanced Systems (ICIAS), (2010) June 15-17, Kuala Lumpur, Malaysia.

[18] X. Cao and S. HastutiKurniawan, "Designing Mobile Phone Interface with Children", In CHI'07 Extended Abstracts on Human Factors in Computing Systems, (2007), New York, USA.

[19] Adriaan W. Mors, 'Context-Aware Route Planning. Multiagent System Technologies Lecture Notes in Computer Science”, Springer Berlin Heidelberg, vol. 6251, (2010), pp. 138-149.

[20] O. Mazhelis, I. Žliobaite and M. Pechenizkiy, "Context-Aware Personal Route Recognitipn", Discovery Science Lecture Notes in Computer Science Volume, (2011) October 5-7, Espoo, Finland.

[21] J. Karat, "The evolution of user-centered focus in the human-computer interaction field" IBM Systems Journal, vol. 42, no. 4, (2003).

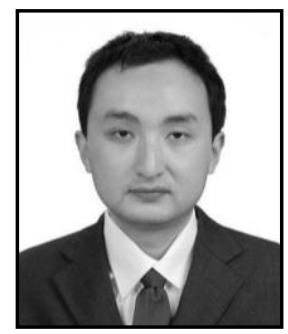

JiangFan Feng, He received his B.S. degree from Southwest Agricultural University, and his $\mathrm{Ph} . \mathrm{D}$ degree from Nanjing Normal University, in 2002 and 2007. He works as associate professor of Chongqing University of Posts and Telecommunications. His main research area include spatial information integration and multimedia geographical information system.
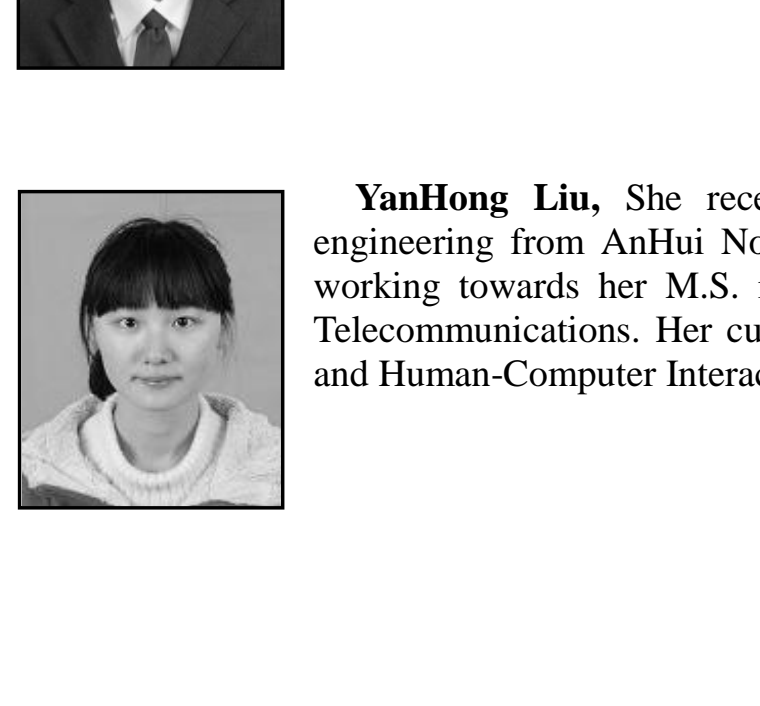

YanHong Ard, She received the B.S. degree in communication engineering from AnHui Normal University, in 2012. She is currently working Kowards her M.S. in the Chongqing University of Posts and Telecommunications. Her current research interests include mobile GIS and Human-Computer Interaction. 
International Journal of Multimedia and Ubiquitous Engineering Vol.9, No.6 (2014)

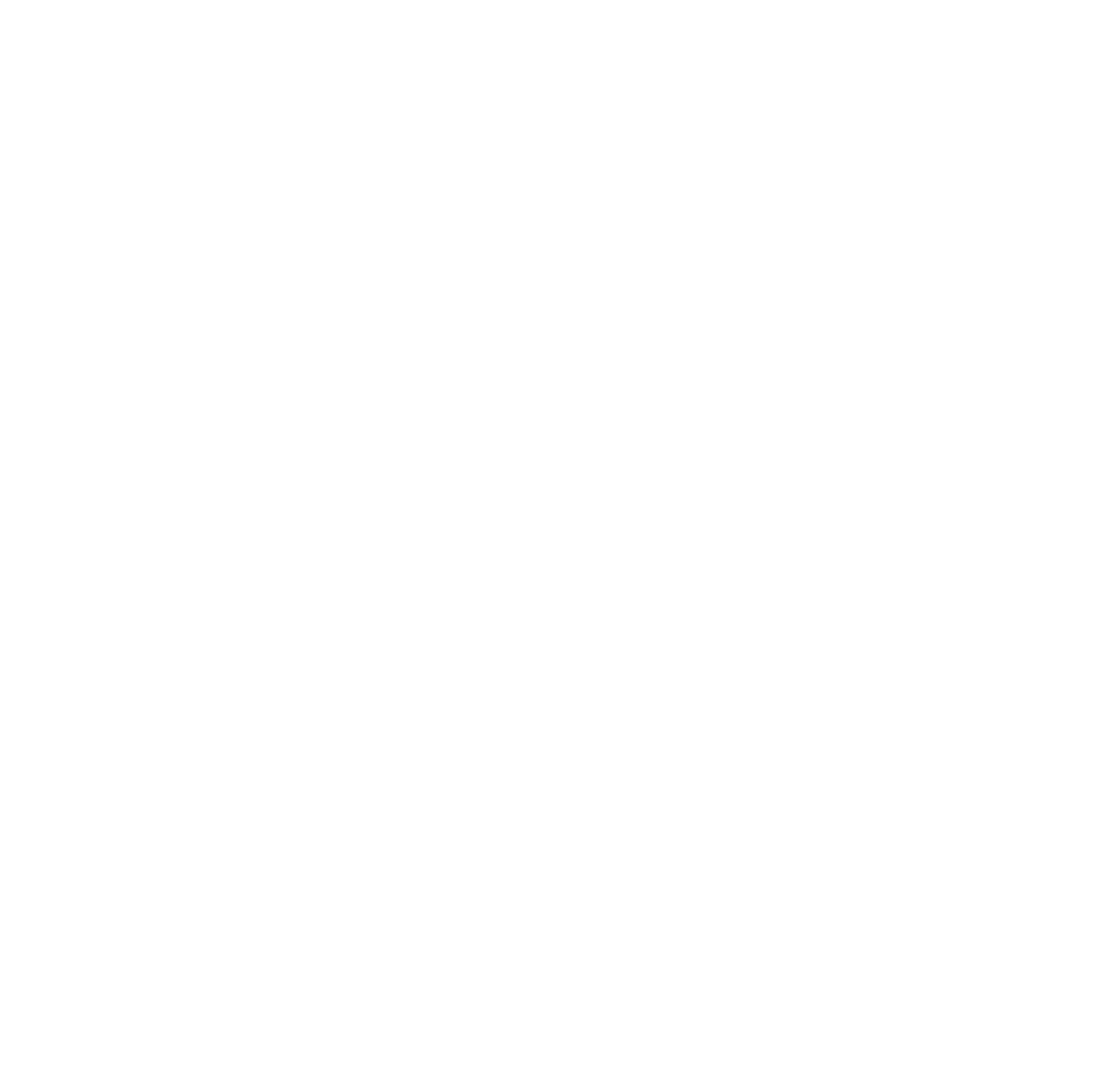

